\title{
Refactoring the B-factor: intuitively extracting structural dynamics from macromolecular disorder
}

\author{
N.M. Pearce ${ }^{1}$, P. Gros ${ }^{2}$ \\ ${ }^{1}$ Free University of Amsterdam, The Netherlands, ${ }^{2}$ Utrecht University, The Netherlands \\ n.m.pearce@vu.nl
}

Displacement parameters (B-factors) play a crucial role in macromolecular structure determination yet are rarely used for biological interpretation. This is somewhat egregious since they account for the local flexibility of individual protein states/conformations. We have developed a new approach [1] for dividing the disorder information in a macromolecular model into a hierarchical series of components on different length-scales, which reveals the components of the atomic disorder that result from molecular disorder, domain disorder, or local atomic disorder. This makes both molecular and atomic disorder intuitively understandable in terms of likely domain motions and internal atomic motions. We demonstrate this new approach by studying the flexibility of the catalytic site in crystal structures of the SARS-CoV-2 main protease. Additionally, we apply the method to structures determined by cryo-EM, where we can investigate and visualize the flexibility in both the extended and non-extended receptor-binding domains of the SARS-COV-2 spike glycoprotein, and in the iron-reductase STEAP4, which hint at a mechanism for electron transfer.

[1] Pearce, N.M., Gros, P., (2021). BioRXiv, https://doi.org/10.1101/2021.03.22.436387.

Keywords: Structural biology; structural dynamics; disorder analysis; macromolecular structures 